\title{
Editorial \\ Thrombosis prophylaxis with new anticoagulants: Routine and specific risk groups
}

\author{
Sibylle A. Kozek-Langenecker \\ Department of Anaesthesia and Intensive Care, Evangelisches Krankenhaus Vienna, Vienna, Austria
}

During the last years, new classes of anticoagulants have entered the market. The direct mechanism of factor Xa and thrombin inhibition offers high anticoagulatory efficacy with reductions in thrombotic events. Improvements in overall quality of care can, however, only be realized when clinicians know pharmacological details of the new drugs and gained experience in daily routine. In the first chapter of this core area volume Professor Samama explains pharmacokinetic determinants of new anticoagulants and summarizes relevant studies on efficacy and safety which formed the basis for official approval.

Professor Spannagl delineates in his chapter means of drug-monitoring which may permit quantification of the actual pharmacodynamic effects of new anticoagulants. Although manufacturers recommend the use of new anticoagulants without specific laboratory monitoring, this may be required in selected patients with impaired drug metabolism, relevant comorbidities and co-medication increasing the risk for the diametric events of thrombosis or bleeding.

The rate of bleeding complications is always a relevant safety issue of anticoagulants. The bleeding risk of new anticoagulants is in the focus in the contribution dedicated to their perioperative use. Anaesthesiologists aim for optimizing the patient's haemostatic system in order to keep the surgical field "dry" which facilitates surgical repair and thereby improves safety. Awareness of bleeding complications resulting from regional anaesthetic techniques culminated in the production of guidelines on the use of nerve blocks in patients on

Correspondence: Sibylle A. Kozek-Langenecker, M.D., Univ.-Doz. and Chairwoman, Department of Anaesthesia and Intensive Care, Evangelisches Krankenhaus Wien, Hans-Sachs-Gasse 10-12, 1180 Vienna, Austria.

Fax: ++43-1-40422-4005, E-mail: sibylle.kozek@aon.at anticoagulants. I will summarize the Austrian and the European recommendations in this chapter.

International and interdisciplinary guidelines regulate thrombosis prophylaxis in many patient groups but critically ill patients are underrepresented. In his chapter Professor Fries elaborates on the repertoire of pharmacological and non-pharmacological thrombosis prophylaxis. Limitations of the traditional indirect coagulation factor inhibition by heparins and subcutaneous anticoagulant administration in intensive care patients will be highlighted. The parenteral direct thrombin inhibitor argatroban enriched intensive care medicine with an efficient drug; the empirical dosefinding process linked theory of drug metabolism and laboratory drug-monitoring to practice of individualized dose-requirements.

Thrombotic events are not restricted to adults also paediatric patients are at risk. Professor Streif accentuates differences in haemostasis in children compared to adults and discusses potential benefits of new anticoagulants in the young population. Dose extrapolations are being proposed and the prevention of heparin-induced thrombocytopenia is emphasized.

Prevention of thrombotic manifestations and bleeding in patients on new anticoagulants is a multidisciplinary challenge. The present core area of this volume pictures the perspective of authors from various medical disciplines and may promote an interdisciplinary management approach as a matter of principle.

\section{Conflict of interest}

The author received honoraria as a speaker from Glaxo Smith Kline, Mitsubishi Pharma and SanofiAventis. She is in the advisory committees of Pfizer/ BMS. 